\title{
Cantidad y distribución de otorrinolaringólogos en los sectores público y privado de salud en Chile
}

\author{
Quantity and distribution of otolaryngologists in public \\ and private health sectors in Chile
}

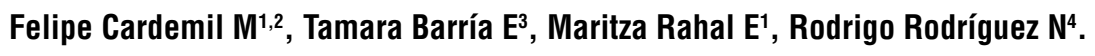

\begin{abstract}
RESUMEN
Introducción: Constantemente se menciona que existe un déficit de otorrinolaringólogos en Chile. Se desconoce la distribución entre el sector público y privado de salud.

objetivo: Determinar la cantidad y distribución de los otorrinolaringólogos que trabajan en el sector público y privado de salud en Chile.

Material y método: Estudio de corte transversal para el que se usaron registros del Ministerio de Salud, Sociedad Chilena de Otorrinolaringología, Superintendencia de Salud y del Departamento de Estadísticas e Información en Salud.

Resultados: Se aprecia que hay 152 especialistas que trabajan en el sector público, en contraste con los 405 totales del país, correspondiendo a 37,5\% de los profesionales que trabajan en el sector público. Al evaluar el total de otorrinolaringólogos registrados a nivel nacional y la población total se obtiene una relación de 1 otorrinolaringólogo/42.589 habitantes, mientras que la relación de otorrinolaringólogos que trabajan en sector público con población beneficiaria de FONASA es de 1 otorrinolaringólogo/85.162 habitantes.

Discusión: El principal problema en Chile no es la cantidad de otorrinolaringólogos, sino su distribución, la que es muy asimétrica. Esto es considerando sólo el número de otorrinolaringólogos, no su carga horaria, ya que al evaluar esta relación probablemente la asimetría aumentaría aún más. Es necesario fomentar la permanencia de los especialistas en el sector público.
\end{abstract}

Palabras clave: Otorrinolaringólogos, distribución, sector público de salud.

\begin{abstract}
Introduction: Constantly there are mentions that there is a shortage of otolaryngologists in Chile. It is unknown the reality of the distribution between public and private health sector.

Aim: The objective of this study was to determine the amount and distribution of otolaryngologists working in the public and private health sector in Chile.
\end{abstract}

\footnotetext{
Servicio de Otorrinolaringología, Hospital Barros Luco Trudeau. Universidad de Chile.

Programa de Doctorado en Salud Pública. Escuela de Salud Pública, Universidad de Chile.

Médico Cirujano. Magíster en Bioestadística, Escuela de Salud Pública, Universidad de Chile.

Médico Cirujano. División de Gestión y Desarrollo de las Personas, Ministerio de Salud, Chile.
} 
Material and method: A cross-sectional study that used the records of the Ministry of Health, Chilean Society of Otolaryngology, Superintendent of Health, and Department of Health Statistics and Information.

Results: There are 152 specialists working in the public sector, in contrast to the 405 total in the country, corresponding to $37,5 \%$ of total professionals working in the public sector. In assessing the relationship between total otolaryngologists in the country with the total population, and otolaryngologists working in the public sector and the beneficiary population of FONASA, there is a relationship of 1 ORL/42.589 and 1 ORL/85.162 inhabitants, respectively.

Discussion: The main problem in Chile is not the amount of otolaryngologists, but its distribution, which is very asymmetric between the private and public sector. It is necessary to promote the retention of specialists in the public sector.

Key words: Otolaryngologists, distribution, public health sector.

\section{INTRODUCCIÓN}

Constantemente se menciona el déficit de médicos especialistas en Chile ${ }^{1}$. Se considera que en este problema influyen factores dependientes del sistema de salud, de los centros formadores, y de los propios médicos². Se estima que en el país existen 29.996 médicos, de acuerdo a datos reportados en el Estudio de Brechas de Oferta y Demanda de Médicos Especialistas en Chile de $2010^{3}$, en el que se aprecia que $73 \%$ de ellos se concentra en la zona central, y que $44 \%$ trabaja en el sector público de salud, con un promedio de 36,8 horas semanales por médico ${ }^{3}$. Además, se ha reportado una relación de 0,2 médicos generales y 0,49 médicos especialistas por cada mil personas 4 .

Entre los años 2005 y 2008, la proporción de médicos generales se redujo desde $19 \%$ a $15 \%$, y la de especialistas certificados por CONACEM aumentó en este período. Sin embargo, esto no se reflejó en una mayor cantidad de especialistas vinculados al sector público, aumentando hacia el sector privado $0^{4-10}$. Actualmente este sector recibe más de la mitad de la producción de médicos, siendo en su mayoría especialistas ${ }^{8}$. La oferta total de horas médicas muestra un total de 289.524 horas de especialistas en el país (6.580 med/eq, o relación de médicos equivalentes a tiempo completo, es decir, 6.580 cargos de $44 \mathrm{hrs})^{11,12}$. La demanda en horas de especialidad y médicos 44 hrs/eq, en relación a la oferta contratada, mostró una importante brecha a nivel nacional, que alcanza en promedio a $30 \%{ }^{12}$.

Las especialidades primarias registradas el 2004 abarcaron 97.015 horas semanales, dentro de éstas las que presentan una mayor cantidad de horas contratadas son anestesiología, traumatología y ortopedia, y neurología y neurocirugía, mientras que en otorrinolaringología 4.351 horas contratadas, 99 médicos en $44 \mathrm{hrs} / \mathrm{eq}(4,5 \%)^{12}$.

En un estudio realizado en España el 2009, adjudican mayor importancia a las desigualdades esporádicas en la distribución geográfica de especialistas en otorrinolaringología que a un déficit real absoluto ${ }^{4}$. En Europa existe un registro del número de especialistas en otorrinolaringología por habitantes, destacando dentro de los países de referencia habitual, los siguientes ${ }^{13}$ (expresado como un otorrinolaringólogo/habitante): Grecia: 1/9.000, Alemania: 1/16.000, España: $1 /$ 20.000, Francia: 1/23.000, Turquía: 1/27.000, Rusia: $1 /$ 38.000, Irlanda 1/100.000, Reino Unido: 1/116.000.

En España, se han publicado diversos conceptos acerca de la oferta y demanda de médicos especialistas, evaluando la variabilidad de tasas poblacionales de especialistas empleados en centros públicos. Dentro de los indicadores demográficos por especialidad para otorrinolaringología en el año 2006 había un total de 1.779 médicos otorrinolaringólogos, donde 1.582 estaban a tiempo completo, con $3,4 \%$ que trabajaba en la red pública, siendo el $58 \%$ de los médicos menores de 51 años, con una tasa de 4,01 ORL/100.000 habitantes, proyectándose una oferta de otorrinolaringólogos en España al 2030 de 1.584 especialistas $^{14}$.

\section{OBJETIVO}

El objetivo del presente estudio fue determinar la cantidad y distribución de los otorrinolaringólogos 
que trabajan en el sector público y privado de salud en Chile, y las características de éstos.

\section{MATERIAL Y MÉTODO}

Se realizó un estudio de corte transversal para el que se utilizó una base de datos del Ministerio de Salud (MINSAL) que consideraba todos los cargos médicos de otorrinolaringólogos (ORL) a noviembre del año 2011. Además, se consideró la cantidad de ORL que figuraban inscritos en la Superintendencia de Salud (SIS), y el listado de ORL miembros de la Sociedad Chilena de Otorrinolaringología, Medicina y Cirugía de Cabeza y Cuello (SOCHIORL). Se incluyeron todos los médicos que trabajaban en servicios de ORL del país, la cantidad de horas semanales, sexo y edad, datos que fueron solicitados a la División de Gestión y Desarrollo de las Personas del MINSAL. Los datos sobre la cantidad de población asignada se obtuvieron desde el Instituo Nacional de Estadísticas para el caso de población general, y del Departamento de Estadísticas e Información en Salud (DEIS) para población inscrita en hospitales de servicios de salud.

Para el análisis se utilizó estadística descriptiva, con frecuencias absolutas y relativas, y promedio y desviación estándar (DE) en caso de variables cuantitativas. Se presentan además las razones entre $\mathrm{ORL}$ y habitantes.

\section{RESULTADOS}

Al evaluar los registros disponibles en el MINSAL hay 152 ORL contratados con contrato en el sector público, los que tenían un promedio de edad de 48 \pm 11 años. El $57,2 \%$ (87 médicos) son menores de 50 años. En cuanto a la distribución por género el $20,1 \%$ del total de ORL contratados eran mujeres.

Según los registros de la SOCHIORL al 2012, existirían 352 especialistas miembros de la sociedad. De éstos, 64 especialistas pertenecen sólo a la SOCHIORL, sin registro en la SIS. Por otro lado, en la SIS se encuentran registrados 346 ORL, apreciándose 53 especialistas no registrados en la SOCHIORL, correspondiendo a $13,1 \%$ de no afiliación. Esto da un total de $405 \mathrm{ORL}$ registrados en el país, correspondiendo esto a 97 mujeres $(24 \%)$ y 308 hombres (76\%). Del total, sólo el 37\% mantiene algún tipo de contrato con el servicio público.

\section{Otorrinolaringólogos por regiones}

Al evaluar por regiones, la que tiene mayor porcentaje de $\mathrm{ORL}$ en el servicio público es la Región Metropolitana (RM), con 59 médicos ( $38,8 \%$ de los ORL contratados en el servicio público del país) seguida en segundo lugar la región del Bío-Bío con $20,4 \%$ (31 especialistas). De la misma manera, si se evalúa cómo se distribuye la carga horaria por región, se observa que dichas regiones son las que poseen mayor cantidad de horas contratadas de ORL con un porcentaje de $32 \%$ y $20,5 \%$, respectivamente.

Al evaluar el número de habitantes totales de la región y la cantidad total que estaría asignado por cada ORL contratado en el servicio público a nivel regional, se aprecia que aquellas con mejor relación ORL/habitantes son las regiones de Bío-Bío, Los Ríos y Magallanes (1/66.098, 1/76.140 y 1/79.600, respectivamente), mientras que las que presentan peor relación ORL/habitantes son las regiones de Atacama, O'Higgins y del Maule (1/282.600, 1/445.900, 1/507.900, respectivamente), ubicándose la RM en el décimo lugar con una proporción de 1/117.722 habitantes.

Respecto a la relación entre ORL y población beneficiaria FONASA por región al año 2011, se aprecia que aquellas regiones con mejor relación son la región de Bío-Bío, de Magallanes, y de los Ríos, con una proporción de 1/53.668 beneficiarios, $1 / 56.862$ beneficiarios y $1 / 65.264$ beneficiarios, respectivamente. Mientras que las regiones con peor relación son las regiones de Atacama, Valparaíso, 0'Higgins y del Maule (Tabla 1).

Al revisar el número de $\mathrm{ORL}$ por región registrado en la SOCHIORL y el MINSAL hasta el año 2011, las regiones con mayor cantidad de $\mathrm{ORL}$ son las regiones Metropolitana, de Valparaíso y del Bío-Bío, siendo a su vez aquellas con mejor proporción de ORL/habitante $(1 / 34.215,1 / 59.250$ y $1 / 62.091$, respectivamente) y las con peor proporción son las regiones de la Araucanía, de O'Higgins y del Maule (1/122.300, 1/ 127.400, $1 / 145.114$, respectivamente) (Tabla 2).

Si se evalúa el tiempo de ORL disponible por población a nivel regional, la región que tiene más horas semanales contratadas de ORL es la RM, seguida por la de Bío-Bío (1.254 horas y 803 horas respectivamente), correspondiendo al $32 \%$ y $20,5 \%$ de las horas contratadas de ORL a nivel país. Mientras que aquellas regiones con menor cantidad de horas semanales disponibles son las de Atacama, Aysén y 
Tabla 1. Cantidad de otorrinolaringólogos que trabajan en sector público por población beneficiaria FONASA por región

\begin{tabular}{|llccc|}
\hline Región & Nombre región & $\begin{array}{c}\text { ORL por región que } \\
\text { trabajan en sector público }\end{array}$ & $\begin{array}{c}\text { Población beneficiaria } \\
\text { FONASA por Región }\end{array}$ & $\begin{array}{c}\text { Relación de 1 ORL /habitante } \\
\text { beneficiario FONASA }\end{array}$ \\
\hline XV & Arica y Parinacota & 2 & 167.584 & $1 / 83.792$ \\
II & Iquique & 3 & 216.800 & $1 / 72.267$ \\
II & Antofagasata & 5 & 346.663 & $1 / 69.333$ \\
III & Atacama & 1 & 231.570 & $1 / 231.570$ \\
IV & Coquimbo & 8 & 577.966 & $1 / 72.246$ \\
V & Valparaíso & 10 & 1.389 .745 & $1 / 138.975$ \\
VI & O'Higgins & 2 & 693.547 & $1 / 346.774$ \\
VII & Maule & 2 & 871.339 & $1 / 435.670$ \\
VIII & Bío Bío & 31 & 1.663 .720 & $1 / 53.668$ \\
IX & Araucanía & 8 & 770.444 & $1 / 96.306$ \\
X & Los Lagos & 9 & 689.683 & $1 / 76.632$ \\
XIV & Los Ríos & 5 & 326.318 & $1 / 65.264$ \\
XI & Aysén & 1 & 71.497 & $1 / 71.497$ \\
XII & Magallanes & 2 & 113.724 & $1 / 56.862$ \\
XIII & Metropolitana & 59 & 4.814 .048 & $1 / 81.594$ \\
Total & Nacional & 152 & 12.944 .648 & $1 / 85.162$ \\
\hline
\end{tabular}

Tabla 2. Cantidad de otorrinolaringólogos que trabajan en sector público y privado por población total por región

\begin{tabular}{|llcrr|}
\hline Región & Nombre de Región & $\begin{array}{c}\text { ORL por región que trabajan } \\
\text { en sector público y privado }\end{array}$ & $\begin{array}{c}\text { Población total por } \\
\text { región a 2011 }\end{array}$ & $\begin{array}{c}\text { Relación de 1 ORL/habitante } \\
\text { Total }\end{array}$ \\
\hline XV & Arica y Parinacota & 2 & 183.200 & $1 / 91.600$ \\
I & Iquique & 3 & 321.700 & $1 / 107.233$ \\
II & Antofagasata & 7 & 581.700 & $1 / 83.100$ \\
III & Atacama & 3 & 282.600 & $1 / 94.200$ \\
IV & Coquimbo & 8 & 728.900 & $1 / 91.113$ \\
V & Valparaíso & 30 & 1.777 .500 & $1 / 59.250$ \\
VI & O'Higgins & 7 & 891.800 & $1 / 127.400$ \\
VII & Maule & 7 & 1.015 .800 & $1 / 145.114$ \\
VIII & Bío Bío & 33 & 2.049 .000 & $1 / 62.091$ \\
IX & Araucanía & 8 & 978.400 & $1 / 122.300$ \\
X & Los Lagos & 9 & 846.600 & $1 / 94.067$ \\
XIV & Los Ríos & 5 & 380.700 & $1 / 76.140$ \\
XI & Aysén & 1 & 105.900 & $1 / 105.900$ \\
XII & Magallanes & 2 & 159.200 & $1 / 79.600$ \\
XIII & Metropolitana & 203 & 6.945 .600 & $1 / 34.215$ \\
Total & Nacional & 328 & 17.248 .600 & $1 / 52.587$ \\
\hline
\end{tabular}

Nota: Se consideró la distribución de 328 otorrinolaringólogos debido a que la SOCHIORL disponía de la localización geográfica de estos y no de la totalidad (352 de ORL registrados).

Magallanes, con 33 horas semanales en total cada una de ellas, lo que correspondería al $0,84 \%$ de las horas contratadas a nivel país.

Según los registros de ORL en la SOCHIORL versus ORL contratados en el servicio público, se aprecia que las regiones en las cuales la mayoría de los ORL no trabajan en el sector público son la región Metropolitana (RM), de O'Higgins, del Maule y de Valparaíso.

Al evaluar la cantidad de horas laborales de ORL contratadas por región para la población beneficiaria FONASA, considerando la proporción de 11 hrs. sema- nales/beneficiarios, se observa que las regiones con mayor tiempo disponible son las de la Araucanía, Aysén, Iquique, Bío-Bío y Coquimbo, mientras que las con peor relación son las regiones de O'Higgins, Atacama y Maule (Tabla 3).

\section{Otorrinolaringólogos por Servicio de Salud (SS)}

En cuanto a distribución de ORL por SS, los que presentan mayor cantidad son los Servicios Metropolitano Oriente, Metropolitano Sur y 
Tabla 3. Cantidad de equivalentes 11 horas otorrinolaringólogo semanales disponibles en sector público por población beneficiaria FONASA por región

\begin{tabular}{|llccc|}
\hline Región & Nombre región & $\begin{array}{c}\text { Población beneficiaria } \\
\text { FONASA por región }\end{array}$ & $\begin{array}{c}\text { Número } 11 \text { hrs/semanales } \\
\text { de ORL contratadas }\end{array}$ & $\begin{array}{c}\text { Población beneficiaria } \\
\text { FONASA asignada para } \\
11 \mathrm{hrs} \text { laborales de ORL }\end{array}$ \\
\hline XV & Arica y Parinacota & 167.584 & 5 & $11 \mathrm{hrs} / 33.517$ \\
I & Iquique & 216.800 & 9 & $11 \mathrm{hrs} / 24.089$ \\
II & Antofagasta & 346.663 & 9 & $11 \mathrm{hrs} / 38.518$ \\
III & Atacama & 231.570 & 3 & $11 \mathrm{hrs} / 77.190$ \\
IV & Coquimbo & 577.966 & 23 & $11 \mathrm{hrs} / 25.129$ \\
V & Valparaíso & 1.389 .745 & 23 & $11 \mathrm{hrs} / 60.424$ \\
VI & O'Higgins & 693.547 & 6 & $11 \mathrm{hrs} / 115.591$ \\
VII & Maule & 871.339 & 13 & $11 \mathrm{hrs} / 67.026$ \\
VIII & Bío Bío & 1.663 .720 & 73 & $11 \mathrm{hrs} / 22.791$ \\
IX & Araucanía & 770.444 & 42 & $11 \mathrm{hrs} / 18.344$ \\
X & Los Lagos & 689.683 & 19 & $11 \mathrm{hrs} / 36.299$ \\
XIV & Los Ríos & 326.318 & 11 & $11 \mathrm{hrs} / 29.665$ \\
XI & Aysén & 71.497 & 3 & $11 \mathrm{hrs} / 23.832$ \\
XII & Magallanes & 113.724 & 3 & $11 \mathrm{hrs} / 37.908$ \\
XIII & Metropolitana & 4.814 .048 & 114 & $11 \mathrm{hrs} / 42.229$ \\
Total & Nacional & 12.944 .648 & 356 & $11 \mathrm{hrs} / 36.361$ \\
\hline
\end{tabular}

Metropolitano Occidente. Mientras que los que poseen sólo un ORL son los servicios de Atacama, Viña del Mar y Arauco. Se aprecia que el Servicio de Salud Metropolitano Oriente y el de Concepción poseen la mayor cantidad de cargos de $\mathrm{ORL}$ contratados por servicio (Tablas 4 y 5 ).

Al evaluar la distribución por sexo en los SS, se observó que sólo en el Servicio Metropolitano Sur existe un predominio de especialistas mujeres (54\%).
Al considerar la población inscrita como beneficiaria FONASA en los SS se observa que los que poseen peor proporción de ORL para la población beneficiaria son: Viña del Mar-Quillota, Libertador B. O'Higgins y Atacama (1/771.260, 1/346.774 y $1 /$ 231.570 , respectivamente) y aquellos con mejor proporción son: Concepción, Bío-Bío Los Angeles, Metropolitano Oriente y Talcahuano (1/33.822, 1/37.317, 1/44.310, 1/49.323, respectivamente) (Tabla 6).

Tabla 4. Servicios de salud con mayor cantidad de médicos por cargo

\begin{tabular}{|lccccc|}
\hline Servicio de salud & Cargos 11 hrs & Cargos 22 hrs & Cargos 33 hrs & Cargos 44 hrs & Total cargos \\
\hline SSMOriente & 5 & 5 & 3 & 1 & 14 \\
SSMSur & 1 & 11 & 1 & 0 & 13 \\
SSMOccidente & 6 & 5 & 1 & 0 & 12 \\
SSConcepción & 2 & 8 & 4 & 0 & 14 \\
SSVSA & 3 & 3 & 3 & 0 & 9 \\
\hline
\end{tabular}

SSMOriente: Servicio de Salud Metropolitano Oriente. SSMSur: Servicio de Salud Metropolitano Sur. SSMOccidente: Servicio de Salud Metropolitano Occidente. SSConcepción: Servicio de Salud Concepción. SSVSA: Servicio de Salud Valparaíso-San Antonio.

Tabla 5. Servicios de salud con menor cantidad de médicos por cargo

\begin{tabular}{|lccccc|}
\hline Servicio & Cargos 11 hrs & Cargos 22 hrs & Cargos 33 hrs & Cargos 44 hrs & Total cargos \\
\hline SSAtacama & 0 & 0 & 1 & 0 & 1 \\
SSVQ & 0 & 1 & 0 & 0 & 1 \\
SSArauco & 0 & 0 & 0 & 1 & 1 \\
SSAysén & 0 & 0 & 1 & 0 & 1 \\
SSChiloé & 0 & 0 & 1 & 0 & 1 \\
\hline
\end{tabular}

SSAtacama: Servicio de Salud de Atacama. SSVQ: Servicio de Salud de Viña del Mar y Quillota. SSArauco: Servicio de Salud de Arauco. SSAysén: Servicio de Salud de Aysén. SSChiloé: Servicio de Salud de Chiloé. 
Tabla 6. Cantidad de otorrinolaringólogos que trabajan en sector público por población beneficiaria FONASA por servicio de salud y equivalentes 11 horas otorrinolaringólogo semanales disponibles en sector público por población beneficiaria FONASA por servicio de salud

\begin{tabular}{|c|c|c|c|c|c|c|}
\hline Servicio de Salud & $\begin{array}{l}\text { Población beneficiaria } \\
\text { FONASA por servicio }\end{array}$ & $\begin{array}{l}\text { ORL por Servicio } \\
\text { de salud }\end{array}$ & $\begin{array}{c}\text { Relación } 1 \text { ORL/ } \\
\text { beneficiario FONASA } \\
\text { de Servicio de salud }\end{array}$ & $\begin{array}{l}\text { Horas contratadas } \\
\text { de ORL por } \\
\text { Servicio de salud }\end{array}$ & $\begin{array}{c}N^{0} \text { períodos } \\
11 \text { hrs laborales/ } \\
\text { semanales de ORL } \\
\text { por Servicio de salud }\end{array}$ & $\begin{array}{c}\text { Relación población } \\
\text { FONASA por } \\
\text { cargo } 11 \mathrm{hrs} \\
\text { (11 hrs/beneficiario) }\end{array}$ \\
\hline Arica & 167.584 & 2 & $1 / 83.792$ & 55 & 5 & $11 \mathrm{hrs} / 33.517$ \\
\hline Iquique & 216.800 & 3 & $1 / 72.267$ & 99 & 9 & $11 \mathrm{hrs} / 24.089$ \\
\hline Antofagasta & 346.663 & 5 & $1 / 69.333$ & 99 & 9 & $11 \mathrm{hrs} / 38.518$ \\
\hline Atacama & 231.570 & 1 & $1 / 231.570$ & 33 & 3 & $11 \mathrm{hrs} / 77.190$ \\
\hline Coquimbo & 577.966 & 8 & $1 / 72.246$ & 253 & 23 & $11 \mathrm{hrs} / 25.129$ \\
\hline Viña del Mar - Quillota & 771.260 & 1 & $1 / 771.260$ & 22 & 2 & $11 \mathrm{hrs} / 385.630$ \\
\hline Valparaíso - San Antonio & 403.148 & 6 & $1 / 67.191$ & 154 & 14 & $11 \mathrm{hrs} / 28.796$ \\
\hline Aconcagua & 215.337 & 3 & $1 / 71.779$ & 77 & 7 & $11 \mathrm{hrs} / 30.762$ \\
\hline Libertador B. O'Higgins & 693.547 & 2 & $1 / 346.774$ & 66 & 6 & $11 \mathrm{hrs} / 115.591$ \\
\hline Maule & 871.339 & 6 & $1 / 145.223$ & 143 & 13 & $11 \mathrm{hrs} / 67.026$ \\
\hline Concepción & 473.503 & 14 & 1/33.822 & 352 & 32 & $11 \mathrm{hrs} / 14.797$ \\
\hline Talcahuano & 295.937 & 6 & $1 / 49.323$ & 143 & 13 & $11 \mathrm{hrs} / 22.764$ \\
\hline Arauco & 146.768 & 1 & $1 / 146.768$ & 44 & 4 & $11 \mathrm{hrs} / 36.692$ \\
\hline Bío-Bío - Los Angeles & 335.851 & 6 & $1 / 37.317$ & 165 & 15 & $11 \mathrm{hrs} / 22.390$ \\
\hline Ñuble & 411.661 & 4 & $1 / 102.915$ & 99 & 9 & $11 \mathrm{hrs} / 45.740$ \\
\hline Araucanía Sur & 594.859 & 6 & $1 / 99.143$ & 231 & 21 & $11 \mathrm{hrs} / 28.327$ \\
\hline Araucanía Norte & 175.585 & 2 & $1 / 87.793$ & 231 & 21 & $11 \mathrm{hrs} / 8.361$ \\
\hline Del Reloncaví & 340.418 & 4 & $1 / 85.105$ & 77 & 7 & $11 \mathrm{hrs} / 48.631$ \\
\hline Chiloé & 146.471 & 1 & $1 / 146.471$ & 33 & 3 & $11 \mathrm{hrs} / 48.824$ \\
\hline Osorno & 202.794 & 4 & $1 / 50.699$ & 99 & 9 & $11 \mathrm{hrs} / 22.533$ \\
\hline Valdivia & 326.318 & 5 & $1 / 65.264$ & 121 & 11 & $11 \mathrm{hrs} / 29.665$ \\
\hline Aysén & 71.497 & 1 & $1 / 71.497$ & 33 & 3 & $11 \mathrm{hrs} / 23.832$ \\
\hline Magallanes & 113.724 & 2 & $1 / 56.862$ & 33 & 3 & $11 \mathrm{hrs} / 37.908$ \\
\hline Metropolitano Central & 619.248 & 5 & $1 / 123.850$ & 99 & 9 & $11 \mathrm{hrs} / 68.805$ \\
\hline Metropolitano Norte & 602.982 & 6 & $1 / 100.497$ & 121 & 11 & $11 \mathrm{hrs} / 54.817$ \\
\hline Metropolitano Oriente & 620.346 & 14 & $1 / 44.310$ & 308 & 28 & $11 \mathrm{hrs} / 22.155$ \\
\hline Metropolitano Sur Oriente & 1.080 .266 & 8 & $1 / 135.033$ & 165 & 15 & $11 \mathrm{hrs} / 72.018$ \\
\hline Metropolitano Sur & 912.180 & 13 & $1 / 70.168$ & 319 & 29 & $11 \mathrm{hrs} / 31.455$ \\
\hline Metropolitano Occidente & 979.026 & 13 & $1 / 75.310$ & 242 & 22 & $11 \mathrm{hrs} / 44.501$ \\
\hline
\end{tabular}

Tabla 7. Cantidad de cargos de otorrinolaringología contratados y distribución de acuerdo al número de horas semanales en el sector público a nivel nacional

\begin{tabular}{|ccr|}
\hline Horas contrato & Frecuencia & Porcentaje \\
\hline 11 & 55 & 31,61 \\
22 & 82 & 47,13 \\
33 & 31 & 17,82 \\
44 & 6 & 3,45 \\
Total & 174 & 100,00 \\
\hline
\end{tabular}

Nota: Son 174 cargos correspondientes a 152 médicos otorrinolaringólogos contratados.

En cuanto al tiempo semanal de ORL contratado por SS, los con menos horas disponibles son Viña del Mar-Quillota, Chiloé, Magallanes, Atacama y Aysén (con 22, 33, 33, 33 y 33 horas/SS, respectivamente), mientras que los servicios con mayor cantidad de horas contratadas serían Metropolitano Sur, Metropolitano Oriente y Concepción, con 319, 308 y 352 horas/ SS. Al evaluar la cantidad de horas laborales de ORL contratadas por SS para la población beneficiaria FONASA, considerando la proporción de 11 hrs sema- nales/beneficiarios, se observa que los servicios con mejor relación son los de Araucanía Norte, Concepción y Metropolitano Oriente, mientras que las con peor relación son los SS Viña del Mar-Quillota, Libertador B. O'Higgins y Atacama (Tabla 6).

\section{Otorrinolaringólogos a nivel nacional}

Los cargos existentes en el servicio público para ORL (174 cargos), el 47,13\% son de 22 horas semanales ( $h /$ 
s), $31,61 \%$ son de $11 \mathrm{~h} / \mathrm{s}, 17,82 \%$ son de $33 \mathrm{~h} /$ s y $3,45 \%$ de $44 \mathrm{~h} / \mathrm{s}$ (Tabla 7). El promedio de horas semanales asignadas es de 21,24 horas $( \pm 8,8 \mathrm{~h} / \mathrm{s})$. Independiente del sexo, los cargos principalmente asignados son de 11 y $22 \mathrm{~h} / \mathrm{s}$, y no hay cargo de $44 \mathrm{~h} / \mathrm{s}$ asignados a mujeres.

Al evaluar la población estimada de habitantes en Chile en el año 2011 (17.248.600 habitantes), y la cantidad de ORL registrados en la SOCHIORL al año 2012, se aprecia una proporción global de 1 ORL/ 49.002 habitantes, lo que aumenta a 1 ORL/42.589 habitantes al considerar al total de ORL registrados en todas las instituciones evaluadas. Al considerar sólo los ORL que trabajan en el sector público y su población FONASA beneficiaria, se aprecia una relación de 10RL/ 85.162 habitantes, lo que equivale a $11 \mathrm{hrs}$ semanales de atención ORL por cada 36.361 habitantes (Tabla 6). Al considerar la cantidad de ORL que trabajan en el sector público por el total de población se aprecia una relación de 1 ORL/112.736 habitantes.

\section{DISCUSIÓN}

La relación de los ORL ajustada por habitante es menor a la recomendación para satisfacer las demandas. En este sentido, el Estudio de Brechas de Oferta y Demanda de Médicos Especialistas en Chile reporta que la razón de médicos ORL con desempeño en el sector público por cada 100.000 habitantes en España y por cada 100.000 beneficiarios de FONASA en Chile fue 3,64 y $0,65^{3}$. Si aplicamos esa misma escala a toda la población chilena, considerando a los que trabajan en el sector privado, la razón es de 2,35. Es decir, en el sector privado, nos parecemos más a un país como España, en términos de cobertura, y en el sector público distamos mucho de esa relación.

En relación a aspectos demográficos, entre el $40 \%$ $49 \%$ de todos los ORL del país tienen más de 50 años ${ }^{3}$, lo que es levemente superior en términos de distribución al promedio de 45,7 años de los que trabajan en el sector público. Respecto a la distribución geográfica, un estudio que incluyó una muestra del $76 \%$ de los médicos reporta que el 8,9\% de los ORL se encuentra en la zona norte, el $69,63 \%$ en la zona central, el 16,75\% en la zona centro-sur, y el $4,71 \%$ en la zona sur ${ }^{15}$. Este mismo informe plantea que la mejor relación entre médicos ORL y 100.000 habitantes se observa en la zona central, con 0,26 especialistas por cada 100.000 habitantes, en contraste con la zona norte, centro-sur y sur que son bastante similares, con $0,17,0,19$, y 0,17 respectivamente ${ }^{15}$. En este sentido, se debe considerar que es probable que el problema de distribución no sea exclusivamente debido a razones económicas, sino también a aspectos geográficos y de incentivos.

Existe una clara asimetría en la distribución de los ORL entre el sector público y privado, esto considerando sólo cantidad de profesionales; probablemente, esta diferencia sería mucho mayor si se considerara el equivalente de médicos a tiempo completo, es decir, la cantidad de horas trabajadas. De esta manera, al ser el promedio nacional de los 152 que trabajan en el sector público de 22 horas/semanales, y asumiendo que trabajan otras 22 horas semanales en el sector privado, este tiempo se sumaría al de los 253 que trabajan exclusivamente en el sector privado, lo que aumentaría la diferencia de manera considerable. Lamentablemente, para conseguir el dato exacto de tiempo trabajado en el sector privado por cada ORL sería necesario realizar un censo nacional de ORL.

Estos datos orientan a que el problema real del "déficit" de especialistas ORL en Chile no es por un déficit absoluto de médicos, es decir, por poca cantidad, sino que se debe principalmente a un problema de distribución, lo que se ha observado en otros contextos médicos ${ }^{16}$. En este sentido, se puede seguir aumentando la formación de especialistas, pero si continúa la fuga de éstos hacia el sector privado, siempre existirá un déficit relativo en el sector público. De hecho, de acuerdo a cifras del MINSAL del año 2011, existía un vínculo de retorno a trabajar al sector público luego de finalizada la especialidad de $33 \%$ del total de médicos especialistas en formación ese año, cifra que era $35 \%$ para los de $\mathrm{ORL}^{2}$, número que probablemente aumentará con la implementación del programa FORDIR (2011) ${ }^{17} 0$ actual CONE (2012), con el que a partir del concurso 2012 existe un vínculo para la formación de ORL con la Universidad de Chile. Impresiona que si mejorara la distribución entre el sector privado y el público, disminuiría la brecha de necesidad de especialistas en el sector público, haciéndose menos evidente la falencia de especialistas. Si por otro lado se decidiera aumentar desmesuradamente el número de cupos de especialización, traería otras desventajas, como que al aumentar la cantidad de médicos en formación y no aumentar las instalaciones necesarias (más horas docentes, mayor número de pabellones, más instrumental, etc.), es probable que disminuya la calidad de la formación de los futuros especialistas, lo que podría 
generar a largo plazo una disminución en el nivel y competencias de la especialidad en nuestro país.

\section{CONCLUSIÓN}

Como se puede observar, el reto de ajustar oferta y demanda de médicos dinámicamente implica tomar decisiones correctas en el momento adecuado sobre el número de plazas educativas, normativa sobre retención y retiro de los médicos en ejercicio, políticas de inmigración, asegurar una distribución geográfica equilibrada, y tomar las decisiones sobre las condiciones de trabajo y sobre los métodos de remuneración. Esto es una problemática compleja, y la complejidad se agrava por la precariedad de los datos ${ }^{14}$. Para corregir esto, se debe comenzar por describir la situación actual de nuestro país. En este sentido, los resultados actuales son poco alentadores y presentan las limitaciones esperables de este tipo de estudios, pero es la mejor evidencia disponible en la actualidad. Sin embargo, si queremos que en Chile se mejore la salud de los más vulnerables y se disminuyan las inequidades asociadas a ésta, es necesario considerar, como individuos y como colectivo, el aporte que los médicos especialistas pueden hacer desde su vocación al servicio del país.

\section{BIBLIOGRAFÍA}

1. Ministerio de Salud, Universidades y Colegio Médico de Chile. Proyecto-Programa de política de formación de especialistas en Chile. Abril 2010.

2. Román 0, Feliú M, Echavarría L. Formación de médicos especialistas en el Sistema de Servicios de Salud de Chile. Su evolución en el período 2007-2010. Rev Méd Chile 2011; 139: 762-9.

3. Estudio de Brechas de Oferta y Demanda de Médicos Especialistas en Chile. Colaboración técnica Banco Mundial - Gobierno de Chile. Serie Cuadernos de Redes № 31. Ministerio de Salud, Chile.

4. Sánchez S, SuÁrez C, Cobeta I. Demanda y oferta de otorrinolaringólogos basada en la evidencia: ¿cuál es el número necesario de especialistas que se deben formar? Acta Otorrinolaringol Esp 2009; 60(6): 443-50.
5. Bastías G, Marshall G, Zúñiga D, Mena B. Número de médicos en Chile: estimaciones, proyecciones y comparación internacional. Rev Méd Chile 2000; 128 (10): 1167-76.

6. Carbonell R, Pérez F, Jiménez F, Lozano I. Estudio de la demanda asistenclal especializada en otorrinolaringología. Rev San Hig Pub 1994; 6: 8(4): 493-502.

7. Goıc A. Disponibilidad de médicos en Chile y su proyección a mediano plazo. Cinco años después. Rev Méd Chile 1999; 127 (10): 1183-8.

8. Román 0. Evolución de la oferta de médicos. Chile 1993-2008. Rev Méd Chile 2010; 138: 22-8.

9. Organización Mundial de la Salud. Informe sobre la salud en el mundo. Colaboremos por la salud. 2006. [Disponible en www.who.int/whr/2006].

10. Organización Panamericana de la Salud. Foro debate ¿Cuántos médicos y enfermeras necesita un país? 2007 [Disponible en http//health-workers.paho.org.].

11. Román 0, Pineda S, Señoret M. Perfil y número de médicos generales que requiere el país. Rev Méd Chile 2007; 135: 1209-15.

12. Román 0, Señoret M. Estado actual de las especialidades médicas en Chile: realidad en el sistema público no municipalizado. Rev Méd Chile 2008; 136: 99-106.

13. Mollenhauer. Trainee exchange program, Manpower of ENTs in Europe 2004. Union europeenne des medecins specialistes. Minutes of the annual meeting of the uemsorl section and board Nice, 22 $2^{\text {nd }}-24^{\text {th }}$ October 2004.

14. González B, Barber B. Oferta y Necesidad de Médicos Especialistas en España (2006-2030). Grupo de Investigación en Economía de la Salud. Universidad de Las Palmas de GC. Marzo 2007.

15. Guillou M, Carabantes J, Bustos V. Disponibilidad de médicos y especialistas en Chile. Rev Méd Chile 2011; 139: 559-70.

16. Salazar C, Cardemil F, Peña J. Estado Actual e Implicancias de la Acreditación de Escuelas de Medicina en Chile. Rev Méd Chile2009; 137: 1126-7.

17. MINSAL. Programas de Formación de Especialistas 2012. [Disponible en http:// www.minsal.gob.cl/portal/docs/1/5741072.pdf. Revisado el 8 de agosto de 2012]. 